\title{
SERVIÇO DE REFERÊNCIA VIRTUAL VIA CHAT: UMA ANÁLISE COMPARATIVA DE SOFTWARES PARA SOLUÇÕES DE CHAT
}

\author{
SERVICIO DE REFERENCIA VIRTUAL A TRAVÉS DE \\ CHAT: UN ANÁLISIS COMPARATIVO DE SOFTWARE \\ PARA SOLUCIONES DE CHAT
}

\author{
Natalia Nakano* \\ Maria José Vicentini Jorente ${ }^{* *}$ \\ Mariana Cantisani Padua***
}

\begin{abstract}
RESUMO:
Introdução: Por volta de 2000, as TIC possibilitaram às bibliotecas universitárias a disponibilização do Serviço de Referência Virtual (SRV), um serviço mediado por software para a interação via chat entre o bibliotecário e os sujeitos em busca de informações de acervos ou de serviços das bibliotecas.

Objetivo: O objetivo da pesquisa que fundamenta este artigo foi comparar os softwares utilizados por instituições diversas para disponibilizar o SRV

Metodologia: As metodologias para a avaliação e construção teórica que subsidiaram este artigo consistiram-se da combinação de um aporte feito a partir da literatura produzida nacional e internacionalmente sobre o assunto, somado à pesquisa exploratória realizada em ambientes digitais para a análise do design dos software de chat, adicionadas à observação participante.

Resultados: Os resultados apresentam e comparam os recursos e características dos softwares analisados.

Conclusões: As conclusões deste estudo apontam para decisões contextuais das instituições e de seus profissionais da informação, de acordo com necessidades e perfis específicos dos sujeitos que interagem com a informação nos ambientes tradicionais e digito-virtuais delineados por parâmetros únicos em cada instituição.
\end{abstract}

Palavras-chave: Informação e tecnologia. Design da informação. Serviço de referência virtual (SRV). Complexidade.

*Doutoranda do Programa de Pós-Graduação em Ciência da Informação da Faculdade de Filosofia e Ciências - UNESP _ Marília. E-mail natinakano@gmail.com

**Docente do Programa de Pós-Graduação em Ciência da Informação da Faculdade de Filosofia e Ciências - UNESP - Marília. E-mail mjjorente@yahoo.com.br

***Doutoranda do Programa de Pós-Graduação em Ciência da Informação da Faculdade de Filosofia e Ciências - UNESP - Marília. E-mail mariana.cantisani@gmail.com 


\section{INTRODUÇÃO}

Os adventos da Internet, e nela da Web, e sua vertiginosa viralidade nos países desenvolvidos e em desenvolvimento estimularam instituições custodiadoras de informações a reformular as formas de disponibilização dos seus serviços, focando em especial os nativos digitais (NAKANO; JORENTE, 2013).

AS bibliotecas universitárias norte americanas, entre essas instituições, viram no início dos anos 2000 a oportunidade de oferecer Serviços de Referência Virtual (SRV) em seus sites, dentre outros serviços online. O SRV possibilitava comunicação direta com o bibliotecário via email e, posteriormente, de chats (ou bate-papo) em tempo real (NAKANO; JORENTE, 2013) e de maneira fluída e dinâmica.

Esse cenário de dinamicidade informacional mediada pelo Design de Informação dos software intermediados pelas Tecnologias de Informação e Comunicação (TIC) motivou a pesquisa que determinou como objeto de estudo os processos de implantação de recursos tecnológicos dos SRV utilizados por bibliotecas universitárias norte-americanas e brasileiras e que ora apresenta-se neste artigo. Na pesquisa, foram comparadas as características dos software utilizados para o SRV, se discutiu a sua oferta de recursos, bem como eventuais benefícios na percepção da biblioteca pelas pessoas que experimentaram o SRV.

A análise se deu sob a perspectiva do Design da Informação (DI), uma vez que a interação entre os sujeitos se dá por meio da interface do sistema do SRV, e, portanto existe uma preocupação quanto à forma como as informações são organizadas e estruturadas no ambiente. Compreendido dentro do âmbito do Design, o Design da Informação (DI) pode ser entendido como o processo de criação de espaços informacionais focados nas necessidades dos indivíduos que interagem com os ambientes e seus recursos.

Mais especificamente, o DI se preocupa com os processos desde a concepção da ideia do sistema, passando pelo planejamento, análise e apresentação da interface. Dessa maneira, compreende também o entendimento de uma mensagem, seu conteúdo, linguagem e forma de 
Natalia Nakano, Maria José Vicentini Jorente, Mariana Cantisati Padua

Serviço de referência virtual via chat: uma análise comparativa de softwares para soluções de chat

apresentação da informação a ser comunicada. Portanto, o DI tem importante papel no desenvolvimento de projetos informacionais que objetivam o acesso e a socialização da informação, e a consequente construção do conhecimento. Não se trata apenas do desenvolvimento e entrega de sistemas informacionais, mas sim se os agentes interagirão com o sistema de forma eficiente e satisfatória.

Embora essa investigação tenha se realizado em contexto brasileiro em comparação com o norte-americano, mostra-se importante para todos os países que têm contexto similar ao do Brasil, ou seja, de orçamento reduzido, com poucos funcionários nos centros de informação, e que necessita mostrar produtividade e eficiência. Dessa maneira, o SRV pode se constituir em uma ferramenta que agrega valor à instituição, uma vez que bem utilizado pode aproximar a instituição da comunidade que ela serve.

O SRV constitui-se de serviços oferecidos aos frequentadores das bibliotecas por meio de ambientes e plataformas da Internet como: correio eletrônico, chat e software de mensagens instantâneas, salas de bate papo, blogs, tutoriais, links, Twitter, busca bibliográfica, fale conosco e solicitação de fotocópia. No entanto, a atual pesquisa concentrou-se no SRV via chat, também definido como mensagem instantânea, mensagem síncrona e atendimento online, i.e., quando a interação entre o profissional da informação e o usuário é realizada em tempo real.

O SRV via chat é uma realidade nos Estados Unidos, e também em outros países, motivando estudos e pesquisas para a avaliação do serviço e da percepção dos internautas que interagem por seu intermédio visando aprimoramento, principalmente. Diversos desses estudos comprovaram a eficácia e opção dos estudantes e/ou pesquisadores universitários norteamericanos pelo chat (LUO, 2008; RUPPEL; VECCHIONE, 2012).

Ruppel e Vecchione (2012) apresentaram resultados indicadores de que os alunos universitários esperam atendimento de referência rápido, conveniente e personalizado, independente do meio (pessoal, por chat ou por mensagem de texto). Por outro lado, seus estudos relataram que a boa comunicação com o bibliotecário contribui para a percepção de utilidade e 
Natalia Nakano, Maria José Vicentini Jorente, Mariana Cantisati Padua

Serviço de referência virtual via chat: uma análise comparativa de softwares para soluções de chat

relevância dos serviços prestados pela biblioteca, segundo os entrevistados; e que um bom serviço de referência no momento da necessidade constrói bons relacionamentos entre alunos e bibliotecários, independentemente do meio utilizado. Ruppel e Vecchione (2012) também destacaram razões pelas se prefere em geral não consultar o bibliotecário pessoalmente, explicação possível para o sucesso de SRV por meio de chat nos EUA.

Em uma pesquisa realizada em 1972 na Syracuse University, segundo Katzer \& Swope, 65\% dos alunos já haviam relatado que não gostavam de pedir ajuda ao bibliotecário porque sentiam que sua pergunta era muito simples, não queriam incomodar ou porque estavam insatisfeitos com alguma experiência anterior (RUPPEL; VECCHIONE, 2012). Além disso, na pesquisa de Massey-Bruzio de 1998, alunos da Johns Hopkins University haviam também definido a biblioteca como "muito complicada" e criticado a desatenção dos bibliotecários, deixando aos indivíduos freqüentadores a difícil missão do auto-atendimento em um ambiente especializado (RUPPEL; VECCHIONE, 2012). Em 2007, Robinson e Reid (RUPPEL; VECCHIONE, 2012) listaram a falta de conhecimento dos serviços oferecidos, a vergonha, a timidez e a ansiedade causadas por barreiras mecânicas e afetivas como desencorajadoras para a comunicação com os bibliotecários. Robison e Reid (2007), por outro lado, encontraram que a referência por chat e os serviços online oferecem uma solução para a barreira da ansiedade.

Nota-se pelo estudo de Ruppel e Vecchione (2012) que, mesmo com a passar do tempo, a percepção gerada pela consulta pessoal ao bibliotecário pouco mudara nos EUA. Por outro lado, os universitários que interagiram com pessoas valorizavam esses serviços de referência porque tal interação pode se beneficiar da linguagem corporal, de informações visuais e de oportunidades de esclarecimento pessoal de questionamentos, o que não ocorre no chat, por exemplo. O estudo de 2012 conclui assim que, o futuro do SRV será o da videoconferência.

A partir deste referencial considera-se que as TIC possibilitaram novos canais de comunicação, interação e compartilhamento pela Internet, e que é indispensável explorar essas possibilidades já consolidadas atualmente. 
No Brasil, um menor numero de bibliotecas universitárias, descritas como bem sucedidas pelos autores consultados no sentido de alcançarem os objetivos propostos pelo serviço, disponibilizam o SRV por meio de chat. A Pontifícia Universidade Católica do Rio de Janeiro (PUC-Rio), motivada por iniciativas internacionais bem sucedidas (CARVALHO; MILLMAN, 2008) disponibiliza o serviço desde 2002; cinco bibliotecas de faculdades da Universidade de São Paulo (USP) oferecem SRV por meio de chat desde 2009 (SANTANA et al., 2010); a Biblioteca Central da Universidade Estadual de Londrina (UEL) disponibiliza o atendimento online desde 2011 (LETRARI; ZWARETCH; ZANINELLI, 2012); a Biblioteca da Área de Engenharia e Arquitetura da Universidade Estadual de Campinas (UNICAMP) desde 2011 (FERREIRA et al., 2012).

Destaca-se que todas as iniciativas descritas nestes estudos se realizaram graças a esforços e motivação dos profissionais da informação responsáveis por esses centros, no sentido de agregar benefícios às instituições que representam, por meio de mais um canal de comunicação. Desta maneira, melhoraram a visibilidade das bibliotecas universitárias em consonância com as demandas dos atores dos processos infocomunicacionais.

Propôs-se, pois, a partir desses estudos, questionamentos pertinentes á utilização de SRV como recurso de atendimento online em tempo real: o atendimento online proporciona uma melhor e/ou maior interação com o profissional da informação na biblioteca universitária? $O$ atendimento online em tempo real é apropriado à realidade brasileira? É um recurso que aproxima os estudantes brasileiros das bibliotecas universitárias? Qual é o custo benefício da implantação desse tipo de serviço? É verdadeiro supor que as pessoas querem suas necessidades informacionais respondidas no momento que elas surgem?

A realização da pesquisa que ora se traduz neste artigo se justificou pela hipótese levantada quanto ao provável benefício que as bibliotecas e outros centros de informação poderiam obter a partir de experiências de outras instituições que já tiveram o seu serviço consolidado. Focaram-se as 
Natalia Nakano, Maria José Vicentini Jorente, Mariana Cantisati Padua

Serviço de referência virtual via chat: uma análise comparativa de softwares para soluções de chat

bibliotecas universitárias, porém, outros centros de informação como arquivos e museus dispostos adequar uma implantação de SRV via chat também podem se beneficiar da experiência de outras instituições que já puderam avaliar vantagens e desvantagens dos software e recursos tecnológicos afins.

\section{METODOLOGIA}

Ao aporte teórico feito a partir da literatura produzida nacional e internacionalmente sobre o assunto, somou-se pesquisa exploratória realizada nos sites dos software de chat, bem como observação participante, na qual o observador/pesquisador é parte dos eventos que estão sendo pesquisados. Tal metodologia apresentou oportunidades de percepção de acontecimentos, o que não teria sido possível a um observador estranho, pois permitiu "[...] não apenas a observação de comportamentos, mas também de atitudes, opiniões e sentimentos" (VIANNA, 2003, p. 50).

Bax (2014) defende que a metodologia científica adequada para conduzir pesquisas na área da Ciência da Informação, em especial nas linhas de Tecnologia e Gestão da Informação e do Conhecimento deve ser a Design Science Research (DSR), pois combina a relevância da aplicação prática com o rigor científico demandado pelas pesquisas na Cl. "A DSR envolve construir, investigar, validar e avaliar artefatos tais como: construtos, arcabouços, modelos, métodos e instâncias de sistemas de informação, a fim de resolver novos problemas práticos." (BAX, 2014, p. 3888). O autor traz ainda para seu estudo vários autores da $\mathrm{CI}$ para concluir que utilizando-se o DSR "é possível tratar um problema de pesquisa sem perder de vista a consideração de sua relevância e aplicabilidade, nem o rigor científico necessário à geração de conhecimento novo." (BAX, 2014, p. 3901). Cita e concorda com Vakkari (1994) para quem a $\mathrm{Cl}$, "[...] como ciência social aplicada é, em grande parte, uma ciência de projeto, ou design science; [...]". Ainda de acordo com o autor, o tema foi recentemente renomeado de "Design Thinking".

Esse estudo buscou analisar o Design da Informação sob a égide do DSR, de forma que a observação participante foi relevante para observar 
questões práticas que os profissionais enfrentam no cotidiano em interação com os sujeitos e com o artefato tecnológico (software)

A observação participante em território nacional foi aprovada pelo Comitê de Ética da UNESP, Campus de Marília, sob o número 2013/636, e se deu presencialmente na Biblioteca Central da Universidade Estadual de Londrina, Paraná, durante os atendimentos online rotineiros, realizados pelos bibliotecários de referência.

A observação participante com bibliotecários norte-americanos se deu digito virtualmente via chat, possibilitando desta forma a observação do atendimento das bibliotecas naquele país quando da interação por meio dos $\mathrm{SRV}$ e questionamentos de referência. Os atendentes dessas bibliotecas foram informados sobre a consulta como procedimento de etapa da pesquisa e como o consentimento das pessoas envolvidas foi obtido informalmente, optou-se pela não reprodução de imagens ou gravações. Por outro lado, quando o software utilizado pela biblioteca permitiu, o histórico dos atendimentos via chat foi enviado por email pelos profissionais consultados.

\section{SOFTWARE PARA SRV VIA CHAT}

Como objetivo deste estudo, apresentou-se, comparou-se e discutiu-se o software de chat utilizado por bibliotecas norte-americanas (LibraryH3/p e Zopim), bem como os utilizados pelas bibliotecas brasileiras atualmente (LiveZilla, Mibew Messenger e Crafty Syntax). Resultante das investigações, o software LibraryH3/p foi escolhido para este artigo, dado o número de bibliotecas universitárias que o utilizam nos EUA: de acordo com informação obtida por meio do website do LibrayH3/p, atualmente mais de 600 bibliotecas e organizações sem fins lucrativos estão utilizando esse software para seus serviços de chat.

No início dos anos 2000, as bibliotecas universitárias já disponibilizavam os SRV por meio de chat nos Estados Unidos (MÁRDERO ARELLANO, 2001, SESSOMS; SESSOMS, 2008). Muitas delas, no entanto, utilizavam software comerciais populares entre os norte-americanos como Yahoo!, MSN, Google Talk e AIM (SESSOMS; SESSOMS, 2008), pois os estudantes tinham seus 
Natalia Nakano, Maria José Vicentini Jorente, Mariana Cantisati Padua

Serviço de referência virtual via chat: uma análise comparativa de softwares para soluções de chat

bibliotecários(as) adicionados(as) às suas listas de contatos nas redes existentes. Verifica-se nesta situação que a página institucional da biblioteca não era acessada para realizar uma consulta, dado que se enviavam mensagens para os bibliotecários em suas listas.

Neste contexto, o software Meebo ${ }^{1}$ tornou-se popular entre as bibliotecas universitárias nos Estados Unidos em 2006 (SESSOMS; SESSOMS, 2008) como parte da sua política de informação. O Meebo possibilitava que uma caixa de diálogo (widget) fosse inserida na página da instituição, ou seja, o internauta acessava a página da biblioteca para entrar em contato com o bibliotecário. Por outro lado, uma comunicação podia ser iniciada com o bibliotecário atendente sem o preenchimento de formulário inicial comum em softwares comerciais de chat. Sob o ponto de vista das bibliotecas, embora o Meebo trouxesse no seu design a limitação de atender apenas uma pessoa por vez, sua utilização evitava o ônus da administração de diferentes sistemas de chat comerciais pelos bibliotecários.

No entanto, outras desvantagens significativas foram apontadas por instituições americanas: o Meebo usa o software Flash que não é acessível para leitores de tela, dificultando o seu acesso à pessoas cegas ou com baixa visão; os links não são clicáveis tornando o copia e cola compulsório nas interações, quando um fechamento acidental pode fechar a página que está sendo usada para o chat (SESSOMS; SESSOMS, 2008). Em 2007, as bibliotecas da University of North Carolina - Chapel Hill e as participantes do projeto Night $O w^{2}$ começaram a utilizar o Pidgin4Lib ${ }^{3}$, solução encontrada por

${ }^{1} \mathrm{O}$ site da Meebo (http://www.meebo.com/) alerta que a Meebo foi adquirida pela Google em 04 de junho de 2012.

2 O Projeto Night Owl é um serviço de referência virtual piloto entre as bibliotecas das universidades da Carolina do Norte em Chapel Hill, North Carolina State, e Duke para usuários que desejam utilizar o serviço depois do horário de atendimento regular de cada biblioteca. A biblioteca da North Carolina State fornece referência virtual para todos os usuários dessas universidades domingos e terças-feiras; Duke às segundas-feiras e a University of North Carolina at Chapel Hill às quartas-feiras.

3 O Pidgin é um programa de chat que permite estar logado em diferentes canais de chat simultaneamente. Assim, uma pessoa pode estar em chat no Messenger, e ao mesmo tempo no Google Talk ou Yahoo chat. O software é mantido por uma comunidade de desenvolvedores, assim é livre e seu código aberto. 
Natalia Nakano, Maria José Vicentini Jorente, Mariana Cantisati Padua

Serviço de referência virtual via chat: uma análise comparativa de softwares para soluções de chat

Eric Sessom no intuito de resolver os problemas causados por aumento do serviço na instituição; o Pidgin4Lib possibilitou de maneira inédita, operadores múltiplos e uma opção de fila de atendimento para cada instituição participante do projeto Night Owl: com esse software tornou-se possível oferecer serviços de chat em tempo real. Todas as instituições envolvidas no Night Owl migraram para o Meebo, e múltiplos bibliotecários podiam a partir daí receber mensagens instantâneas e chats do Meebo sem desconectar outros atendimentos.

O serviço aumentou consideravelmente: de acordo com Sessoms e Sessoms (2008) os atendimentos cresceram em 300\% em comparação com o semestre anterior. No entanto, o Pidgin4Lib estava restrito à sua arquitetura de pares, as atualizações tinham que ser feitas em muitos computadores, em três grandes universidades. Além disso, na opinião dos autores, o software continha bugs difíceis de serem reparados dentro da sua estrutura (SESSOMS; SESSOMS, 2008). Essa foi a motivação para o desenvolvimento do LibraryH3/p, o sistema utilizado correntemente pelas instituições participantes do projeto Night Owl para a disponibilização do SRV de dia e à noite.

\subsection{LibraryH3Ip}

O desenvolvimento independente do LibraryH3lp por Pam Sessoms e Eric Sessoms, levou-os a escolher soluções de software de código aberto ${ }^{4}$ e o protocolo Extensible Messaging and Presence Protocol (XMPP) 5 baseado em Extensible Markup Language (XML).

Como parte dos procedimentos na biblioteca, os bibliotecários responsáveis pelo SRV registram uma conta de administração na página do LibraryH3/p, na qual é possível adicionar múltiplos usuários e filas de atendimento e tarefas como referência, circulação, serviços, etc. as filas de

4 Open Source Software (OSS) são software de computadores cujo código de fontes está disponível e licenciado por uma licença na qual o detentor dos direitos os cede para estudo, mudança e distribuição do software para qualquer pessoa e para qualquer propósito. Frequentemente são desenvolvidos de maneira pública e colaborativa. $<$ http://en. wikipedia.org/w/index.php?title=Open-source software\&oldid=613236526.

5 Esse protocolo foi originalmente nomeado Jabber, e desenvolvido pela comunidade opensource Jabber em 2009 para mensagens instantâneas, informação de status e manutenção de lista de contato. http://en.wikipedia.org/w/index.php?title=XMPP\&oldid=612663575. 
Natalia Nakano, Maria José Vicentini Jorente, Mariana Cantisati Padua

Serviço de referência virtual via chat: uma análise comparativa de softwares para soluções de chat

tarefas aparecem online quando há pelo menos um operador designado para aquela fila e os administradores da conta podem visualizá-la, ao protocolo, a duração do chat, o tempo de espera para outro atendimento e os chats que ficaram sem reposta. Tais scripts podem posteriormente ser apagados ou baixados nos computadores, criando históricos e também é possível armazenar os textos das conversas no servidor do LibraryH3/p.

Quando um internauta inicia um contato, o chat é recebido como uma mensagem na lista de contatos do bibliotecário. Se mais de um bibliotecário daquela fila estiver online, todos receberão a mensagem, mas apenas o que responder primeiro será conectado a esse usuário; os outros serão desconectados e receberão uma mensagem indicando qual bibliotecário assumiu o chat. No LibraryH3/p ainda é possível transferir o chat para outra fila ou bibliotecário, enviar um arquivo para quem requisitou as informações, um script por email ou ainda visualizar chats anteriores desse atendimento. As caixas de diálogo são facilmente manejáveis e propiciam notificações sonoras tanto para o bibliotecário quanto para o internauta em atendimento. Os links enviados nos atendimentos são clicáveis e abrem em novas janelas ou abas.

Além dessas características, pode-se escolher abrir uma janela para controlar o seu tamanho, pois geralmente as caixas de diálogo inseridas nas páginas são pequenas. As janelas são customizáveis e internacionalizadas, possibilitando a visualização do texto na língua preferencial no navegador dos envolvidos na interação. Também é possível customizar a janela quando o serviço estiver offline para o direcionamento para outros serviços disponíveis.

A Figura 1 mostra um exemplo de possível customização.

Figura 1 - Exemplo de customização para períodos de serviço off-line

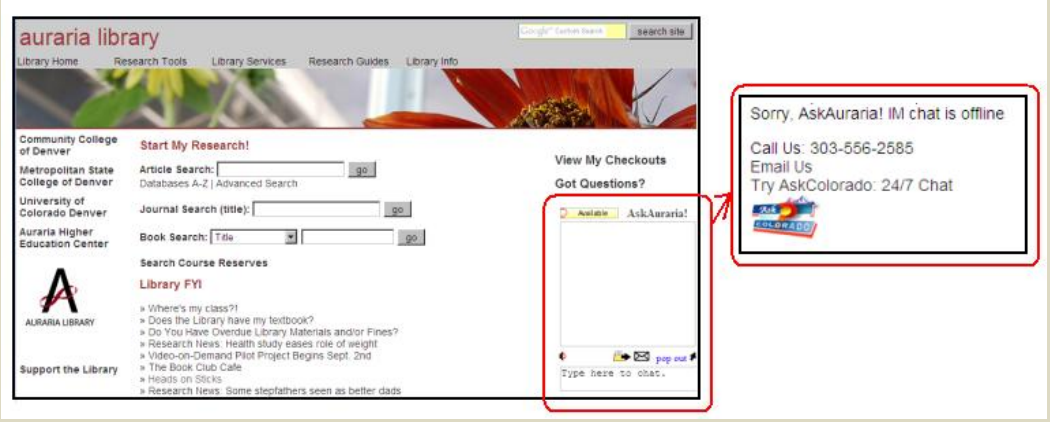

Fonte: Sessoms e Sessoms (2008).

Inf. Inf., Londrina, v. 21, n. 3, p. 124 - 148, set./dez., 2016. www.uel.br/revistas/informacao/ 


\subsection{LiveZilla}

O LibraryH3/p não encontrou aderência no Brasil, onde variadas opções foram adotadas: o LiveZila é um dos software utilizados pelas bibliotecas da Universidade de São Paulo - Biblioteca da Faculdade de Medicina Veterinária e Zootecnia (FMVZ), Escola de Engenharia de São Carlos (EESC), o Instituto de Física de São Carlos (IFSC), o Instituto de Psicologia (IP) e a Faculdade de Odontologia (FO) - e pela Biblioteca Central da Universidade Estadual de Londrina, Paraná (UEL).

Embora a informação publicada na literatura científica sobre o software LiveZilla, restrinja-se aos artigos de Santana et al. (2010) e Nakano e Jorente (2013), é importante destacar que a constituição do LiveZilla como um software de chat online para visitantes de páginas comerciais na internet o caracteriza como uma interface de vendas. Gratuito para habilitar apenas um operador, e pago a partir de dois operadores, os custos aumentam de acordo com o número de operadores, tem características de software proprietário com licenças variadas. Suas características podem ser analisadas de acordo com uma subdivisão em 4 grupos:

1. Características gerais, como base de dados MySQL, customização, editor de tradução, PHP API;

2. Características Help Desk/ Ticket System, como receber emails via POP ou IMAP, enviar e receber anexos, corretor ortográfico, indicador de tempo de espera, alertas e notificações, sistema Ticket System que organiza os emails, chats e ligações em um sistema de bilhetes;

3. Características de chat em tempo real, como encaminhamento de chat para outros operadores, possibilidade de deixar mensagem se o serviço estiver offline, formulário de feedback, transferência de arquivos, tradução automática, envio de email com o texto da conversa; 
4. Características de monitoramento de visitante em tempo real: envio de convites de chat automático (proativo), banir visitante, filtro de segurança, filtros de visitantes baseados no URL e website-pushes;

5. Atualmente, o LiveZilla oferece aplicativos para os sistemas operacionais iOS, Android, Windows Phone e Blackberry. Para a utilização do aplicativo, é necessário que uma versão 5.3.0.6 (ou maior) esteja instalado em um servidor, portanto, não se trata de um software em Beta perpétuo. Entretanto, essa característica permite que o operador (bibliotecário) atenda o serviço por meio de um dispositivo móvel. Além do LiveZilla, outro software utilizado por bibliotecas universitárias no Brasil é o Mibew Messenger.

\subsection{Mibew messenger}

O Mibew Messenger é o software utilizado pela Biblioteca da Área de Engenharia e Arquitetura (BAE) da UNICAMP. A consulta na literatura com a palavra chave Mibew Messenger no Parthenon da UNESP retornou com um artigo publicado em croata. Esse trabalho (ŠTRKALEC; POLONIJO; VODOPIJEVEC, 2013) faz uma comparação entre os software Mibew Messenger, Zopim e o InstanteStore Live Chat. Os autores concluem que o Mibew Messenger é um software adequado e confiável para instituições que necessitem de um produto gratuito para múltiplos operadores e múltiplos atendimentos. A única desvantagem relatada em 2013 foi o fato da comunidade desenvolvedora do Mibew Messenger não ter realizado nenhuma mudança significativa nos dois anosanteriores (ŠTRKALEC; POLONIJO; VODOPIJEVEC, 2013). No entanto, a comunidade de desenvolvedores (GitHub), que mantém o Mibew promovendo suas atualizações, lançou em setembro de 2014 a versão 2.0-alpha3, e em julho de 2015 algumas correções nos códigos foram realizadas e lançada a versão 2.1.06. Assim, o projeto Mibew não está estagnado.

\footnotetext{
${ }^{6}$ https://github.com/Mibew/mibew/releases/tag/v2.1.0.
} 
Natalia Nakano, Maria José Vicentini Jorente, Mariana Cantisati Padua

Serviço de referência virtual via chat: uma análise comparativa de softwares para soluções de chat

O Mibew Messenger é um software desenvolvido para chat que permite assistência um-a-um em tempo real diretamente do website da instituição. É open source e gratuito sob os termos da licença Apache 2.07. Suas características e recursos estão divididos em 5 grupos. O primeiro relaciona-se ao valor do investimento na escolha do software que é de código aberto, livre de custos.

O segundo ponto trata de suas principais características físicas: chat em tempo real, número ilimitado de operadores, visitantes e chats multi línguas, executável no servidor da instituição; botão de chat disponibiliza status online e offline (possui o recurso de mensagem caso o atendente esteja offline); a janela de chat apresenta formulário prévio ao chat opcional, customização da janela, possibilidade de envio de chat por email, alerta sonoro e visual a cada nova mensagem; habilidades do operador: mensagens automáticas, rastreamento de clics para chegar ao seu website, histórico de chat com busca, notificação de mudança de página, visualização dos chats ativos, administrador pode assumir o chat, relatórios estatísticos diários.

A Figura 2 mostra a interface administrador quando o chat está em andamento.

Figura 2 - Interface administrador com chat em andamento

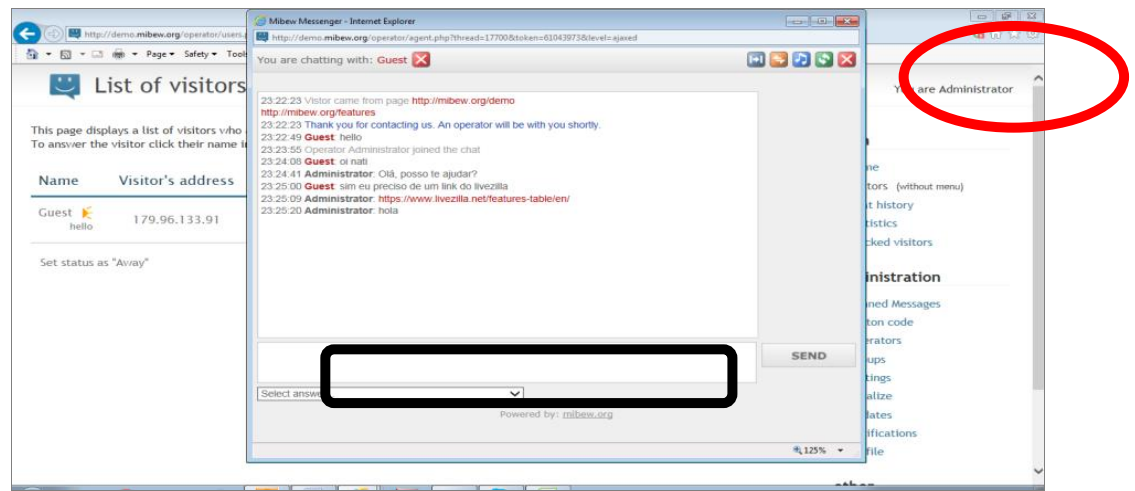

Fonte: Mibew Messenger (2016).

\footnotetext{
${ }^{7}$ A Licença Apache é uma licença para software livre. Exige a Inclusão do aviso de copyright e disclaimer, permite o uso e distribuição do código fonte no software open source quanto no proprietário.
}

Inf. Inf., Londrina, v. 21, n. 3, p. 124 -148, set./dez., 2016. 
Os botões visíveis na janela do atendente possibilitam: transferir o chat para outro operador, ver histórico de chat, ligar e desligar o alerta sonoro, atualizar a página e fechar o chat. No campo "Select answer" o atendente pode escolher enviar mensagens automáticas previamente inseridas.

\subsection{Crafty syntax}

O Crafty Syntax é o software utilizado pela PUC-Rio atualmente. A busca pela palavra chave Crafty Syntax no Parthenon da UNESP, Periódicos CAPES, Scopus, Springer, E-LIS, LISTA e LisZen não retornou com artigos científicos sobre o software.

Trata-se de um produto open source programado em PHP e MySQL e tem três versões: uma versão gratuita, uma versão premium e uma versão avançada. Dentre suas características e recursos, o software permite múltiplos operadores e diferentes línguas, respostas automáticas, visualização da digitalização nas sessões de chat, alerta sonoro, mensagem quando o atendente estiver offline, proatividade, ou seja, o chat pode ser iniciado pelo sistema uma vez habilitado pelo operador.

\subsection{Zopim}

Quando em consulta com o profissional da Biblioteca Alden da Ohio University, foi-nos recomendado um artigo (estudado para discussão na sua biblioteca), que traz uma perspectiva inovadora para o SRV por meio de chat. O artigo Proactive Reference Service (ZHANG; MAYER, 2014) relata a experiência da Biblioteca Grasselli, da Universidade John Carroll em Ohio com o serviço de chat. De acordo com o relato, de fevereiro de 2010 a junho de 2012, a biblioteca registrou um total de 24.864 transações de referência, das quais, $3 \%$ foi realizada por meio de referencia por chat, utilizando o software gratuito Meebo, $9 \%$ por telefone e $88 \%$ pessoalmente. Para a biblioteca, o serviço de chat não estava sendo utilizado eficientemente, e acreditava-se ser devido à característica passiva do software - ou seja, o serviço fica a disposição do usuário inserido na página da biblioteca, mas o bibliotecário de referência entra em atividade apenas quando o chat é acionado pelo usuário. 
Por isso, o serviço com o Meebo foi encerrado em meados de 2012 quando a biblioteca decidiu por uma abordagem proativa para alcançar seus usuários. Para alcançar esse objetivo, adotou-se o software comercial Zopim, que oferece a característica de disparo automático de chat. Acreditava-se que essa característica faria com que os alunos se tornassem cientes do serviço de chat online oferecido pela biblioteca. De acordo com os autores, o Zopim é fácil de instalar, baseado na web (web-based) e pode ser inserido no site da biblioteca.

A janela de chat fica fixa no canto inferior da tela, mesmo que o usuário desloque a barra de rolagem para cima ou para baixo ou mude de página.

É possível estabelecer o tempo de espera até que o chat seja iniciado automaticamente pelo software. No caso da Biblioteca Grasselli, apos 3 minutos de navegação na página da biblioteca, o software abre automaticamente uma janela de ajuda ao internauta com a mensagem "Pergunte-nos".

Quando o internauta responde à questão iniciada automaticamente, o bibliotecário é alertado com a abertura de uma janela e um sinal sonoro. $\mathrm{O}$ bibliotecário então continua o diálogo como se ele próprio tivesse iniciado a conversa. Por meio das URLs que aparecem na tela, o bibliotecário pode visualizar o caminho que a consulta está tomando. Outra característica do software mencionada por Zhang e Mayer (2014) é o Chat Rescuer: quando um usuário inicia uma consulta, mas o bibliotecário não pode atender, depois de 3 minutos a ferramenta notifica o usuário com a seguinte mensagem: "Desculpenos por fazer você esperar. Nossos bibliotecários estão ocupados no momento. Nós o atenderemos assim que possível" (ZHANG; MAYER, 2014, p. 204, tradução nossa).

O serviço com o Zopim foi lançado em outubro de 2012, e graças ao disparo automático de chat, no final de março de 2013, a porcentagem de atendimentos por chat subira de $3 \%$ na avaliação anterior para $21 \%$ enquanto as consultas por telefone caíram de $9 \%$ para $6 \%$ e os atendimentos pessoais caíram de $88 \%$ para $73 \%$. Com relação a comparação quanto à consulta por chat, elas foram divididas em consultas iniciadas pelo sistema Zopim e 
Natalia Nakano, Maria José Vicentini Jorente, Mariana Cantisati Padua

Serviço de referência virtual via chat: uma análise comparativa de softwares para soluções de chat

consultas iniciadas pelo na outra ponta a dos sujeitos que buscam informações. Do total de atendimentos realizados por chat, $70 \%$ foram iniciadas pelo sistema e $30 \%$ foram iniciadas pelo usuário.

Com a análise dos textos de chat, descobriu-se que tipo de perguntas são realizadas por meio do serviço de chat. Elas foram classificadas em 3 categorias: referência/pesquisa; instrucional/técnica/políticas; e outras. Descobriu-se que as questões iniciadas pelos estudantes são, na maioria, para pedir instruções, enquanto que as iniciadas pelo sistema são, na maioria, relacionadas a referência e pesquisa.

Zhang e Mayer concluíram que, embora em algumas ocasiões a oferta de ajuda seja declinada, haja oposições à ideia da janela saltar na tela e interromper pesquisa de maneira inconveniente, a experiência com Zopim é considerada pela biblioteca como positiva, pois acredita-se que perguntas surgem das abordagens do sistema e a biblioteca continuará com o serviço de chat proativo por meio do software (ZHANG; MAYER, 2014).

$\mathrm{Na}$ atualização da pesquisa em 2016, a biblioteca FMVZ da USP mudou a interface inicial da página da biblioteca, passando a utilizar o sistema Wordpress ${ }^{8}$. Um dos recursos desse sistema é a possibilidade de instalar um plugin para atendimento virtual. As características desse sistema, em comparação com as características dos softwares são: pode ser anônimo, ou a instituição pode escolher que o nome e o endereço eletrônico seja requerido; possui versão gratuita e versão pró paga, possibilidade de banir visitante, não tem publicidade, é multilíngue, possibilita acesso de histórico de conversas, é responsivo, é customizável, permite atender multivisitantes ao mesmo tempo, porém com um atendente na versão free.

O sistema WordPress apresenta vantagens e desvantagens. As grandes vantagens são as possibilidades de customização e funcionalidades que o sistema permite, tanto na versão gratuita quanto na versão paga. Porém, a grande desvantagem está no fato de que o sistema de chat depende da

\footnotetext{
8 WordPress é um aplicativo de sistema de gerenciamento de conteúdo para Web. http://br.wordpress.com.
} 
instalação da plataforma WordPress para todo o site da biblioteca, ou seja, se o site da biblioteca ainda não estiver em WordPress, é preciso uma reestruturação completa do site, o que pode não ser permitido pela instituição. Por exemplo, na Unesp o site da biblioteca é institucional, ou seja, utiliza o sistema de gerenciamento de conteúdo que a Unesp disponibiliza. No caso da UFMVZ, percebe-se que utilizam um servidor da USP, porém não utilizam o mesmo sistema de gerenciamento de conteúdo da instituição. Assim, para utilizar o plugin do WordPress, a biblioteca precisa consultar a seção técnica de informática da universidade.

Levantamos que a Faculdade de Odontologia da USP9 não oferece mais o serviço de SRV, o Instituto de Engenharia de São Carlos (EESC) agora usa o Mibew, e o Instituto de Física da USP de São Carlos agora usa o software PHPlive ${ }^{10}$.

Dentre os recursos e características que o software PHPlive disponibiliza são: permite anonimato do usuário, em sua versão de teste/gratuita só pode ser utilizada por 10 dias, para ser utilizado por um maior período necessita da compra; não possui recursos de acessibilidade; permite a inserção de links clicáveis; pode ser operado por múltiplos operadores; não possui código aberto; envia relatórios e dados estatísticos; permite o envio de texto de chat para o usuário; envia alerta sonoro; realiza a troca de arquivos; é multilíngue; possui alguns temas padrões do sistema que permitem a alteração da interface; permite bloquear visitante e possui a funcionalidade de proatividade.

\section{COMPARAÇÃO DAS CARACTERÍSTICAS E RECURSOS DOS SOFTWARE}

A partir da literatura pesquisada (FERREIRA et. al., 2012; LUO, 2008; SANTANA et. al., 2010; SESSOMS; SESSOMS, 2014; ZHANG; MAYER, 2014), as seguintes características e recursos foram elencados como relevantes na escolha de um software: anonimato, gratuidade, recursos de

${ }^{9}$ http://www.fo.usp.br/sdo/?page_id=3043

$10 \mathrm{http}: / /$ www.phplivesupport.com/mobile/?r=1\&key=pi-5-ykq-m 
Natalia Nakano, Maria José Vicentini Jorente, Mariana Cantisati Padua

Serviço de referência virtual via chat: uma análise comparativa de softwares para soluções de chat

acessibilidade para deficientes visuais; links clicáveis; possibilidade de habilitar multi-operadores; ter código aberto; possibilidade de gerar relatórios e dados estatísticos; envio de texto de chat por e-mail; alerta sonoro para mensagens; possibilidade de enviar arquivos; possibilidade de escolha de idioma; customização da janela; recurso de banir ou bloquear visitantes inconvenientes; construção de mensagens automáticas e proatividade.

O Quadro 1 demonstra quais características os software de chat usados pelas bibliotecas estudadas disponibilizam.

Quadro 1 - Características dos software de chat

\begin{tabular}{|c|c|c|c|c|c|}
\hline \multirow{2}{*}{ Recursos e Características } & \multicolumn{5}{|c|}{ Software } \\
\hline & LibraryH3Ip & LiveZilla & Mibew & Crafty Syntax & Zopim \\
\hline Anonimato do usuário & Sim & Não & Sim & Não & Sim \\
\hline Gratuidade & Não & Sim & Sim & Sim & Não \\
\hline Acessibilidade & Sim & Não & Não & Não & Sim \\
\hline Links clicáveis & Sim & Sim & Sim & Sim & Sim \\
\hline Multi operadores & Sim & $\operatorname{Sim}$ & $\operatorname{Sim}$ & Sim & Sim \\
\hline Código aberto & Não & Não & $\operatorname{Sim}$ & Sim & Não \\
\hline Relatório de dados estatísticos & Sim & Sim & Sim & Sim & Sim \\
\hline Envio do texto de chat para o usuário & Sim & $\operatorname{Sim}$ & Não & $\operatorname{Sim}$ & Sim \\
\hline Alerta sonoro & Sim & Sim & Sim & Sim & Sim \\
\hline Troca de arquivos & Sim & $\operatorname{Sim}$ & Não & Não & Sim \\
\hline Opção de linguagem & Sim & $\operatorname{Sim}$ & $\operatorname{Sim}$ & $\operatorname{Sim}$ & Sim \\
\hline Customização & Sim & Sim & Sim & Sim & Sim \\
\hline Banir/Bloquear visitante & Sim & Sim & Sim & Não & Sim \\
\hline Mensagens automáticas & $\operatorname{Sim}$ & $\operatorname{Sim}$ & $\operatorname{Sim}$ & $\operatorname{Sim}$ & Sim \\
\hline Chat proativo & Não & Sim & Não & Sim & Sim \\
\hline
\end{tabular}

Fonte: Elaborado pela autora. 


\section{$5 \quad$ RESULTADOS}

Esse estudo fez um levantamento dos recursos e características consideradas relevantes para um produto de solução de chat sob o ponto de vista da biblioteca e dos bibliotecários. A Figura 5 apresenta um resumo dessas características e recursos, elaborado de acordo com o Quadro 1.

Figura 5 - Sumário dos recursos e características dos software analisados nesse estudo

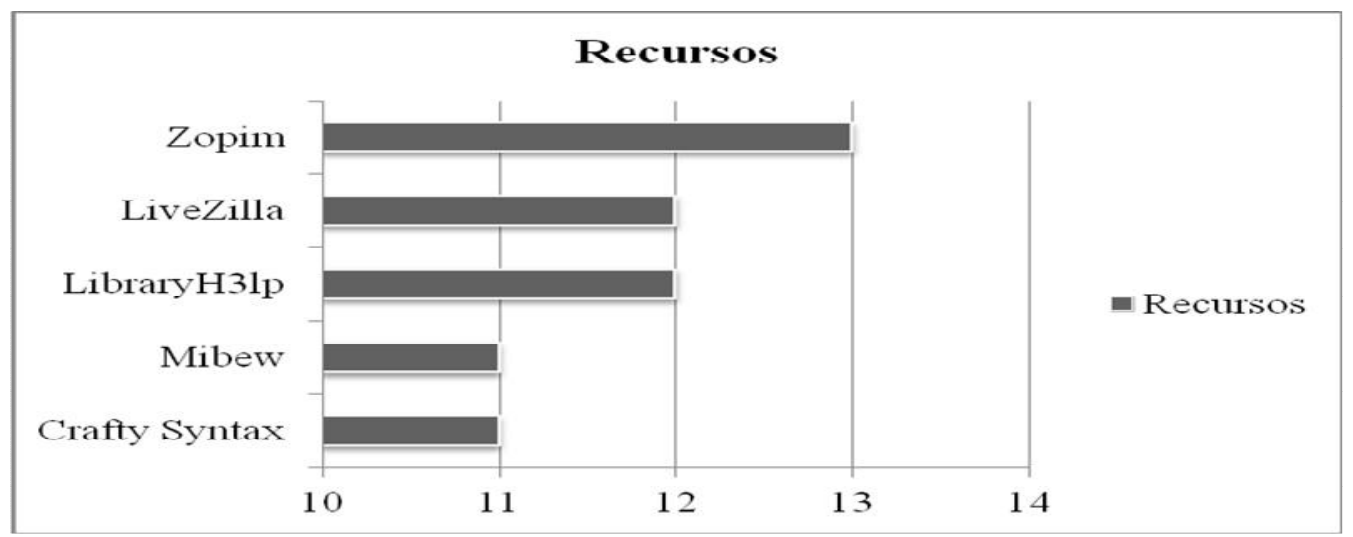

Fonte: Elaborado pela autora.

Embora o software Zopim destaque-se por características e recursos positivos, ele é proprietário e pago, com características progressivas, conforme as funcionalidades contratadas. Já o preço do LibraryH3/p depende do número de alunos da universidade.

Outro recurso que pode ser considerado importante, e que o LibraryH3/p ainda não tem, é o recurso de proatividade. Em comunicação informal com o autor Zhang (ZHANG; MAYER, 2014), após o encerramento do Meebo, o LibraryH3/p foi comparado ao Zopim e esse foi o escolhido justamente por apresentar o recurso de proatividade.

Por outro lado, se a instituição percebe que o preenchimento de formulário com dados antes do início do chat é um fator que pode desmotivar o início ao chat, o LibraryH3/p garante total anonimato, das duas partes envolvidas.

Considerando que as bibliotecas universitárias brasileiras podem ter prioridades anteriores ao investimento em um software para atendimento por 
Natalia Nakano, Maria José Vicentini Jorente, Mariana Cantisati Padua

Serviço de referência virtual via chat: uma análise comparativa de softwares para soluções de chat

chat, e que existe a disponibilidade de outros software confiáveis gratuitamente na rede, pode-se concluir que tanto o LibraryH3/p quanto o Zopim são inviáveis para a realidade da biblioteca universitária brasileira.

O LiveZilla é um software proprietário, e portanto seu código não é aberto. Ele é gratuito na versão para um operador, o que pode ser uma desvantagem para uma instituição que espera tráfego no atendimento; o Mibew Messenger é de código aberto, gratuito e permite múltiplos operadores.

Se a instituição não espera tráfego no atendimento e gostaria de implantar o serviço de referência de chat proativo, o LiveZilla possui o recurso de convite de chat. Quanto ao Crafty Syntax, ele possui esse recurso de proatividade, e embora o usuário não fique anônimo, o formulário requisita apenas o nome.

Sob a perspectiva metodológica aplicada (Design Thinking ou DSR) neste estudo, a exploração dos websites e a observação participante realizada na UEL permitiram constatar que, embora os profissionais desses centros não tenham considerado epistemologicamente o DSR, as equipes das bibliotecas intuitivamente seguiram as fases do Design Thinking (ou DSR) ao implantar o software, o que corrobora o estudo de Bax (2014), no sentido de que a Cl tem praticado o DSR, porém sem se dar conta do rigor científico envolvido na sistematização dos passos tomados: os passos tomados pela equipe de profissionais que refletem o Design Thinking partiram de um problema a ser resolvido: como agregar mais um serviço que pode aproximar a biblioteca da comunidade? As equipes se reuniram e decidiram testar de implantação do SRV. Em seguida, avaliaram o serviço, e concluíram que este traz benefícios para a biblioteca e para a comunidade que ela serve.

O passo seguinte, seguindo a metodologia, seria realizar uma revisão da disponibilização do serviço baseado no feedback da comunidade e partir para resolução do problema que surgir a partir desse feedback, trazendo, dessa maneira, constante melhora para a instituição e para a comunidade servida.

Lancaster (1994, p. 8), em seu artigo Ameaça ou oportunidade? O futuro dos serviços de biblioteca à luz das inovações tecnológicas criticou a posição dos bibliotecários naquela época, com relação à tecnologia: 
Natalia Nakano, Maria José Vicentini Jorente, Mariana Cantisati Padua

Serviço de referência virtual via chat: uma análise comparativa de softwares para soluções de chat

"Esta implicação - que as capacidades tecnológicas é que determinam as características da instituição, ao invés da instituição adotar ou adaptar tecnologias para cumprir seus objetivos e metas - parece, hoje, muito dominante na profissão do bibliotecário".

No entanto, Lancaster (1994), sabiamente concluiu que, embora a tecnologia tenha colocado novas ferramentas dentro da biblioteca, elas nem sempre são usadas com propriedade, pois os bibliotecários confiam demais somente nas tecnologias para resolver os problemas de informação quando, na verdade, inteligência artificial nenhuma pode substituir o talento e intelecto de um bom bibliotecário na busca e acesso à informação.

Nesse mesmo sentido, este estudo recomenda que cada instituição, por meio de seus bibliotecários, decida sobre suas necessidades contextuais e de acordo com o perfil de seus interlocutores, e assim defina quais as características do software de SRV. Dessa maneira, o software ideal para a biblioteca será aquele com mais características positivas, de acordo com esse estudo, ou não.

Com relação aos benefícios que a implantação de um SRV por chat pode trazer à instituição, a observação participante na biblioteca da UEL, no Paraná, concluiu que o SRV aproximou os internautas da biblioteca e poupouos do custo de uma ligação telefônica e também da espera de uma resposta por e-mail.

[...] as bibliotecárias relataram acreditar que a disponibilização do serviço é válida principalmente quando o atendimento é realizado a ex-alunos da universidade que se encontram em outras cidades; ou alunos de pós-graduação que utilizam o serviço estando na sua cidade de origem sem terem que realizar uma ligação à longa distância. Em ambos os casos, os usuários têm sua necessidade informacional resolvida por um profissional da informação que está à disposição na biblioteca (NAKANO; JORENTE, 2014, p. 180).

Além dessa experiência positiva, Santana et al. (2010) relata que o sucesso da implantação do SRV na Faculdade de Medicina Veterinária e Zootecnia da Universidade de São Paulo em 2009 inspirou outras 4 faculdades a seguirem o exemplo: a Escola de Engenharia de São Carlos (EESC), em setembro de 2009; o Instituto de Física de São Carlos (IFSC) e o Instituto de 
Psicologia (IP) em outubro de 2009; e a Faculdade de Odontologia (FO) em dezembro de 2009.

Santana et al. (2010) afirma que - como principais benefícios trazidos pelo SRV para as instituições - os serviços virtuais, de modo geral, têm que estar em consonância com a demanda, e por isso a referência por chat é apropriada; as tecnologias atuais possibilitam a disponibilização do serviço com software gratuitos e de código aberto; o chat está disseminado na comunidade acadêmica; o atendimento feito por telefone traz um custo que pode ser evitado pelo uso da mensagem instantânea; o correio eletrônico pode não atender a necessidade do usuário por não ser síncrono; os serviços baseados em tecnologia trazem benefícios, pois demandam atualização constante do profissional (SANTANA et al., 2010).

Assim, esse artigo concluiu que além de agregar benefícios reais à instituição, sob a perspectiva dos profissionais da informação que trabalham com SRV, o atendimento por chat é apropriado para a realidade brasileira no universo deste estudo, pois propicia o atendimento por meio de uma ferramenta familiar para a comunidade acadêmica, de forma síncrona e com a qualidade do atendimento pessoal.

\section{REFERÊNCIAS}

BAX, Marcello Peixoto. Design science: filosofia da pesquisa em ciência da informação e tecnologia. In: ENCONTRO NACIONAL DE PESQUISA EM CIÊNCIA DA INFORMAÇÃO, 15., 2014, Belo Horizonte. Anais eletrônicos... Belo Horizonte: ANCIB/UFMG, 2014, p. 3883-3903. Disponível em: $<$ http://www.bax.com.br/publications/artigos/design-science-filosofia-dapesquisa-em-ciencia-da-informacao-e-tecnologia>. Acesso em: 30 abr. 2016.

CARVALHO, Pamela Lisboa; MILMAN, S. M. Atendimento ao usuário através de chat: a experiência da biblioteca da PUC-RIO. In: SEMINÁRIO NACIONAL DE BIBLIOTECAS UNIVERSITÁRIAS, 15., 2008, São Paulo. Anais eletrônicos... São Paulo: CRUESP, 2008. Disponível em: $<$ http://www.sbu.unicamp.br/snbu2008/anais/site/pdfs/2841.pdf $>$. Acesso em: 30 abr. 2012. 
FERREIRA, Danielle Thiago et al. Serviço de atendimento on-line por chat: experiência da biblioteca da área de Engenharia e Arquitetura da Unicamp. In: SEMINARIO NACIONAL DE BIBLIOTECAS UNIVERSITÁRIAS, 17., 2012, Gramado. Anais eletrônicos... Porto Alegre: UFRGS, 2012. Disponível em: $<$ http://www.sbu.unicamp.br/seer/ojs/index.php/simtec/article/download/7584/28 60>. Acesso em: 30 abr. 2016.

LANCASTER, Frederick Wilfrid. Ameaça ou oportunidade? O futuro dos serviços de biblioteca à luz das inovações tecnológicas. Revista da Escola de Biblioteconomia da UFMG, Belo Horizonte, v. 23, n. 1, p. 7-27, jan./jun. 1994.

LETRARI, Maria Aparecida Santos; ZWARETCH, Natali Silvana; ZANINELLI, Neide Maria J. Atendimento online por meio do chat: um serviço de referência 2.0 na biblioteca central da UEL. In: SEMINARIO NACIONAL DE BIBLIOTECAS UNIVERSITÁRIAS, 17., 2012, Gramado. Anais... Porto Alegre: UFRGS, 2012.

LUO, Lili. Chat reference evaluation: a framework of perspectives and measures. Reference Services Review, Ann Arbor, v. 36, n. 1, p. 71-85, 2008.

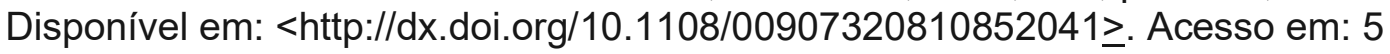
jan. 2012.

MÁRDERO ARELLANO, Miguel Ángel. Serviços de referência virtual. Ciência da Informação, Brasília, v. 30, n. 2, p. 7-15, 2001. Disponível em: <http://www.scielo.br/pdf/ci/v30n2/6206.pdf>. Acesso em: 25 out. 2012. MIBEW Messenger. Disponível em: <http://demo.mibew.org/operator/>. Acesso em: 21 maio 2016.

NAKANO, Natalia; JORENTE, Maria José Vicentini. Serviço de referência virtual: uma análise do serviço de referência síncrono disponibilizado por bibliotecas de universidades brasileiras. In: SEMINARIO CIENTíFICO ARQUIVOLOGIA E BIBLIOTECONOMIA, 3., 2013, Marília. Anais... Marília: UNESP, 2013.

NAKANO, Natalia; JORENTE, Maria José Vicentini. Serviço de referência virtual: implantação do serviço de chat. Informação \& Informação, Londrina, v. 19, n. 1, p. 164-184, jan./abr. 2014.

RUPPEL, Margie; VECCHIONE, Amy. It's research made easier! SMS and chat reference perceptions. Reference Services Review, Ann Arbor, v. 40, n. 3, p. 423-448, 2012.

SANTANA, Anderson et al. Atendimento online em bibliotecas: a experiência da universidade de São Paulo. In: SEMINÁRIO NACIONAL DE BIBLIOTECAS UNIVERSITÁRIAS, 16., Rio de Janeiro. Trabalhos... Rio de Janeiro: UFRJ, 2010. Disponível em: <http://www.gapcongressos.com.br/eventos/z0070/ trabalhos/final_225.pdf>. Acesso em: 30 abr. 2012. 
Natalia Nakano, Maria José Vicentini Jorente, Mariana Cantisati Padua

Serviço de referência virtual via chat: uma análise comparativa de softwares para soluções de chat

SESSOMS, Pam; SESSOMS, Eric. LibraryH3lp: a new flexible chat reference system. Code4lib Journal, Sydney, v. 4, set. 2008. Disponível em: <http://journal.code4lib.org/articles/107>. Acesso em: 17 jan. 2014.

ŠTRKALEC, Tomislav; POLONIJO, Davor; VODOPIJEVEC, Alen. Kako pitati knjipnièara? usporedba programskih rješenja za mrepnu komunikaciju korisnika knjipnice i knjipnièara. Z naših knjibnica, Zagreb, v. 62, n. 7-8, p. 272-275, 2013. Disponivel em: <http://pierre.fkit.hr/hdki/kui/vol62/broj7-8/272.pdf>. Acesso em: 20 jun. 2014.

VAKKARI, Pertti. Library and information science: its content and scope. In: GODDEN, Irene, P. (Org.). Advances in librarianship. New York: Academic Press, 1994. v. 30. p. 1-55.

VIANNA, Heraldo Maecelim. Pesquisa em educação: a observação. Brasília: Plano, 2003.

ZHANG, Jie; MAYER, Nevin J. Proactive chat reference: getting in the users' space. College \& Research Libraries News, Chicago, v. 75 , v. 4, p. 202-205, abr. 2014. Disponível em: <http://crln.acrl.org/content/75/4/202.full>. Acesso em: 2 maio 2014.

\section{Title}

Virtual reference service via chat: a comparative analysis of software for chat solutions

\section{Abstract}

Introduction: In the early 2000s, ICT allowed university libraries to offer Virtual Reference Service, reference service mediated by software to enable interaction via chat between the librarian and the people in search for information in library collections or services.

Objective: The objective of the research that grounds this article was to compare the software used by several institutions to provide Virtual Reference Service.

Metodology: The methodology for evaluation and theoretical construction that supported this article consisted of a study on the theoretical contribution produced nationally and internationally on the subject, in addition to an exploratory research conducted on digital environments to assess the design of the chat software, as well as participant observation.

Results: The results show and compare the features and characteristics of the analyzed software.

Conclusion: The results of this study point to contextual decisions from the institution and the information professionals, according to the needs and profiles of the subjects who interact with the digital environment, outlined by unique parameters in each institution.

Keywords: Information and technology. Virtual reference service. Chat. 
Natalia Nakano, Maria José Vicentini Jorente, Mariana Cantisati Padua

Serviço de referência virtual via chat: uma análise comparativa de softwares para soluções de chat

\section{Titulo}

Servicio de referencia virtual a través de chat: un análisis comparativo de software para soluciones de chat

\section{Resumen}

Introducción: En la década de 2000, las TIC permitió a las bibliotecas universitarias la disponibilidad de Servicio de Referencia Virtual (SRV), un software de servicio mediada por la interacción a través de chat entre el bibliotecario y los temas de las colecciones de información o servicios de la biblioteca.

Objetivo: El objetivo de la investigación que subyace en este artículo fue comparar el software utilizado por diversas instituciones para proporcionar el SRV.

Metodología: Las metodologías para la evaluación y la construcción teórica que apoya este artículo consistió en la combinación de una contribución de la literatura nacional e internacional producen sobre el tema, además de la investigación exploratoria en entornos digitales para el análisis de diseño de software de chat, añadido a la observación participante.

Resultados: Los resultados muestran y comparan los atributos y características de los softwares analizados.

Conclusión: Los resultados de este estudio apuntan a las decisiones contextuales de las instituciones y sus trabajadores de la información, de acuerdo a las necesidades y perfiles de los individuos específicos que interactúan con la información en entornos tradicionales y virtuales dígitos delimitada por los parámetros individuales en cada institución.

Palabras clave: Información y Tecnología. Diseño de información. Servicio de Referencia Virtual (SRV). Complejidad.

Recebido em: 10.04.2016

Aceito em: 18.11.2016 